Research Paper:

\title{
The Effect of Benson Relaxation Technique on the Fatigue Severity of Patients With MS
}

\author{
Saeed Mirhosseini' ${ }^{1}$, Abouzar Mohammadi $^{1}$ (D), Mahboobeh Rezaei $^{2}$ (D), Neda Mirbagher Ajorpaz ${ }^{2 *}$ (D)
}

1. Trauma Nursing Research Center, Kashan University of Medical Sciences, Kashan, Iran.

2. Department of Nursing and Midwifery, Autoimmune Diseases Research Center, Kashan University of Medical Sciences, Kashan, Iran.

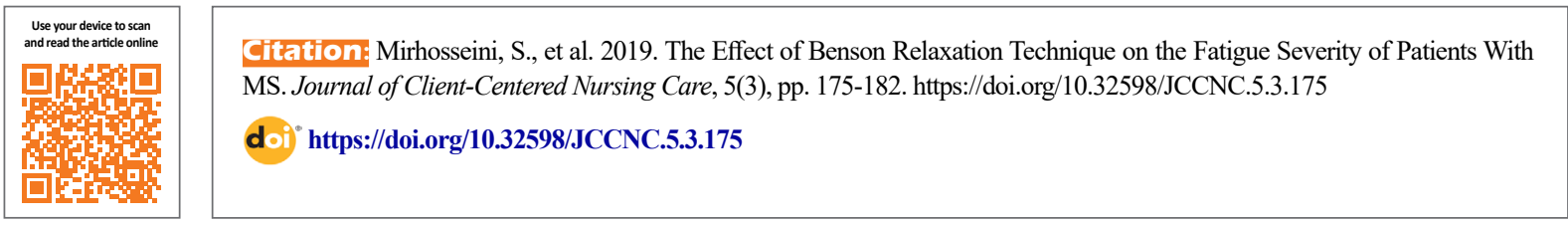

\section{(c) (1) (s)}

Article info:

Received: 03 Jan 2019

Accepted: 07 May 2019

Published: 01 Aug 2019

Keywords:

Benson relaxation technique, Fatigue, Multiple Sclerosis (MS)

\begin{abstract}
A B S T RA C T
Background: Fatigue is a frequent symptom in Multiple Sclerosis (MS) that can interfere with the daily functioning of the patient. The present study aimed to determine the effect of the Benson relaxation technique on the fatigue severity of MS patients.

Methods: This was a clinical trial study. A total of $60 \mathrm{MS}$ patients with $<5$ degrees of disability were enrolled and randomly assigned into two groups ( $\mathrm{n}=30$ per group). The intervention group performed the Benson relaxation technique twice daily, and for 20 minutes during one month. No intervention was provided to the control group. Before and after the study, the fatigue questionnaire was completed by the patients, and the two groups were compared. The obtained data were analyzed in SPSS using the Chi-squared test, Independent Samples t-test, and Paired Samples t-test.
\end{abstract}

Results: Benson relaxation exercises significantly reduced the average fatigue severity of patients with MS $(\mathrm{P} \leq 0.05)$.

Conclusion: Benson relaxation technique can be used as a cost-benefit, safe, and ordinary complementary therapy to reduce fatigue in patients with MS.

\section{* Corresponding Author:}

Neda Mirbagher Ajorpaz, PhD.

Address: Department of Nursing and Midwifery, Autoimmune Diseases Research Center, Kashan University of Medical Sciences, Kashan, Iran. Tel: +98 (36) 155540021

E-mail:mirbagher_n@kaums.ac.ir 


\section{Highlights}

- Fatigue is among the most frequent symptoms associated with MS.

- The relaxation technique is a method of mental relaxation to managing stress, decreasing anxiety, and promoting physical discomfort.

- Benson relaxation exercises significantly reduced the average fatigue severity of patients with MS.

\section{Plain Language Summary}

The current study assessed the effect of Benson relaxation techniques on the fatigue of patients with MS in a hospital in Kashan City, Iran. MS complications affect all aspects of the individual's life and disrupt the normal course of family life due to its debilitating nature. Fatigue is among the most common symptoms associated with MS. Fatigue management, and general health promotion includes pharmaceutical and non-pharmaceutical interventions. Benson relaxation technique can be used as a low-cost, safe, and ordinary complementary therapy to reduce fatigue in patients with MS.

\section{Introduction}

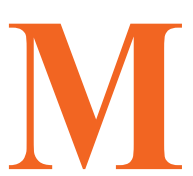

ultiple Sclerosis (MS) is a chronic, non-traumatic, autoimmune, and demyelinating Central Nervous System (CNS) disease (Correale et al. 2017). It is the most prevalent disorder of myelin sheath in the CNS, which occurs in the early stages of a young age or, more generally, in the third and fourth decades of life. It emerges with localized demyelination or myelin fragmentation of the CNS and is accompanied by irregular periods of recovery and relapse of symptoms (Kaminska et al. 2017). MS is diagnosed with recurring symptoms, such as weakness, anorexia, numbness, dizziness, cognitive changes, blindness, and long periods of physical disability (Ontaneda et al. 2017). According to the Atlas of the MS International Federation, the number of MS patients has been increased from 2.1 million in 2008 to 2.3 million in 2013. According to this international organization, the prevalence of MS in the Middle East and North Africa regions, including Iran, is at a low to a moderate level (Montalban et al. 2018). However, recent studies have suggested that the incidence and prevalence of MS is increasing in this region, especially in females (Etemadifar et al. 2016; Etemadifar et al. 2017).

Studies on MS in Iran reevaled that the incidence and prevalence of this disease have risen in the last decade as the MS outbreak ranged from 35.5 per 100000 population in 2006 to 85.8 in 2013 (Etemadifar et al. 2016). MS complications, due to its debilitating nature, affect all aspects of an individual's life, and disrupt the normal course of the family life. Besides, the overwhelming majority of people are youth, and this spreads the problem to a large extent in the community (Etemadifar et al. 2017). Fatigue is among the most frequent symptoms associated with MS, with an incidence rate of $50 \%$ to 93\% (Rooney et al. 2019). Two-thirds of patients with MS suffer from fatigue as one of the three disabling symptoms of their disease (Dayapoglu and Tan 2012).

Fatigue is defined as the subjective deficiency of physical or mental energy, i.e. perceived by the individual or care provider and affects the routine or desired activities (Heine et al. 2016). To manage fatigue and to promote general health, pharmaceutical, and non-pharmaceutical interventions could be used (Rahimimoghadam et al. 2017). Considering the complications associated with pharmacotherapy, applying non-pharmacological methods that can reduce fatigue and improve the general health of these patients sounds logical (Heidari et al. 2017; Rahimimoghadam et al. 2019). Fatigue is often the primary purpose of treatment in the rehabilitation of patients with MS and various non-medical interventions, such as therapeutic exercises and energy conservation courses (Heine et al. 2016).

Non-pharmacological or complementary therapies are often associated with lower side effects and risks that can be used alone or in combination with other methods. One of such methods is relaxation (Abedi et al. 2018). Relaxation is a behavioral intervention that has many types. One of these techniques was developed by Benson (1975) and had a very favorable effect on the complete relaxation of all muscles. Implementing relaxation is cost-effective and feasible. Relaxation is a method of mental calming technique to managing stress, decreas- 
ing anxiety, mood disorders, physical discomfort, and the autonomic dysfunction of the nervous system and affects the quality of sleep (Rambod et al. 2013). Relaxation, by balancing anterior and posterior hypothalamus, decreasing the sympathetic nervous system activity, and the secretion of catecholamine reduces muscle tension and undesirable physiological effects, blood pressure, and pulse rate and muscle spasm, and improves respiration.

In the Benson relaxation technique, when patients relax and expand each muscle by the technique, they can easily relax the whole body and reduce stress and anxiety (Jafari, Janati, et al. 2018). Dayapoğlu and Tan documented that Progressive Muscle Relaxation Technique (PMRT) improves the quality of sleep and fatigue in patients with MS (Dayapoglu \& Tan 2012). Van Kessel et al. also argued that relaxation training reduced fatigue in patients with MS (van Kessel et al. 2008). Several studies have assessed the effect of Benson relaxation technique on other diseases; however, to our knowledge, no research has been carried out using this technique in MS. Most studies on patients with MS have used PMRT technique, i.e. more difficult to learn by the patient than Benson technique, and requires advanced training. Moreover, studies on using relaxation to reduce fatigue in patients with MS in Iran are scarce. Therefore, the current study aimed to determine the effect of Benson relaxation technique on the fatigue severity of patients with MS.

\section{Materials and Methods}

This was a clinical trial study. A total of 60 patients were recruited in Shahid Beheshti Hospital of Kashan, Iran, from 2016 to 2017. Using the Pocock sample size formula (Pocock 1983), the optimal sample size of each group was estimated as 30. According to Etemadifar (2016), d (the difference between the two mean scores) and $\partial$ (standard deviation) were respectively equal to 7.8 and 7 , with a type I error probability of 0.05 and a power of 0.80 . The study participants were randomly assigned to the Benson technique $(n=30)$ and control $(n=30)$ groups (simple allocation using www.random.org).

The research tools included a demographic questionnaire comprising age, gender, job, marital status, and educational level and summarized the fatigue questionnaire of Brief Fatigue Inventory (BFI), including 10 questions.

The first question specifies the presence or absence of unusual fatigue in the last week with yes or no answers. The next questions assess the extent of current fatigue, fatigue in the last 24 hours, the highest rate of fatigue in the last 24 hours, the effect of last 24 hours, fatigue on overall activity, mood, walking ability, communication with others, and the enjoyment of life. Each is measured at a scale of 0-10. A zero score represents the lack of fatigue, and a score of 10 indicates the highest level of fatigue. Ultimately, the patient's overall fatigue comes from the total score of questions from 2-10 (9 questions) and its division into 9. Zero reflects no fatigue, 0.1-3.9 indicates mild fatigue, 4-6.9 represent moderate fatigue, 9-9.9 show severe fatigue, and 10 is a sign of very severe fatigue (Chen et al. 2016). This is a standard scale and has been used in numerous studies. Several studies in Iran have confirmed the reliability of this questionnaire (Niino et al. 2014; Nunnari et al. 2015). Cronbach's alpha coefficient of this scale was calculated as $86 \%$ in the current study.

The study inclusion criteria were as follow: the diagnosis of MS (relapsing-remitting type) by a neurologist, having a record at Shahid Beheshti Medical Center in Kashan, gaining scores between 1 and 5 of the Expanded Disability Status Scale (EDSS) benchmark, being aged between 20 and 45 years, no history of attacks in the last three months, no history of mental conditions, dementia, and intellectual disabilities, and not practicing cognitivebehavioral techniques, such as yoga and meditation for up to 6 months before the intervention.

The study exclusion criteria were as follow: Unwillingness to participate in the study, acute or chronic physical or mental disorders (e.g. hepatotoxic, respiratory, cardiac, skeletal, and renal diseases), The occurrence of any MS complications during the study which prevent the application of Benson relaxation technique and missing more than two training sessions.

The disability status score of all the study patients was estimated based on the disability determination questionnaire. Based on this scale, all patients with a score of $\leq 5$ were selected (Carpinella et al. 2014). Initially, 60 subjects who met the inclusion criteria and agreed to participate in the study were recruited purposively. After presenting the necessary explanations and the study purpose and obtaining informed consent, patients were randomly assigned into two intervention and control groups using a random allocation method. To control confounding factors and to prevent data contamination, 30 subjects were selected in the control group; then, 30 subjects were recruited in the intervention group.

The two groups were matched in terms of diet, medications, and treatment type. The educational content of the sessions included questions and answers about the benefits of relaxation and practical presentation of the 
technique. In these sessions, after explaining how the exercises should be performed, the research units were requested to conduct the exercises in the presence of the researcher to ensure their accuracy. The sessions were held in the room of the patients in the relevant department by separating the patient's bed from the others by a partition and in coordination with the ward's head nurse. The research subjects performed the relaxation technique with the researcher for 20 minutes. The training method was also provided to the research samples along with the educational pamphlet. It included the training process, and they were requested to relax for 20 minutes every day, twice daily (in the morning and evening), for a month, at their home.

The Benson relaxation technique instruction included 5 steps. The study participants were instructed to sit in a relaxed position; close their eyes; relax all the body muscles, beginning from the soles of the feet moving forward up; breath in and out through nose and concentrate on breathing sounds, and silently say the word 'one' after exhalation (Rambod et al. 2013).
Accordingly, in the intervention group, words, such as God, prayer, Quran, and religion, were firstly used according to the belief system of the patient. Based on the literature, these words calm the patient (Mowla et al. 2017). Then, it was suggested that the patient is in a comfortable position, close his/her eyes and relax their muscles; taking a deep breath; then, repeat the prayer that he/she believes in and gives him/her peace of mind, and clear his/her mind. In the control group, the researcher was only present on the patient bedside, and no intervention was performed. The control group received routine care. The training sessions were held during the patients' hospitalization period. The technique continued during the hospitalization and after discharge from the hospital. The study participants' compliance with the Benson relaxation technique was ensured via phone contacts. They were also requested to complete a daily self-report performance sheet. The first author that has a training certificate in this field attended the MS center every day for the participants' performance follow-up and reinforcements and collected their daily self-report performance sheets. The first author's cell phone num-

Table 1. The demographic data of the study participants

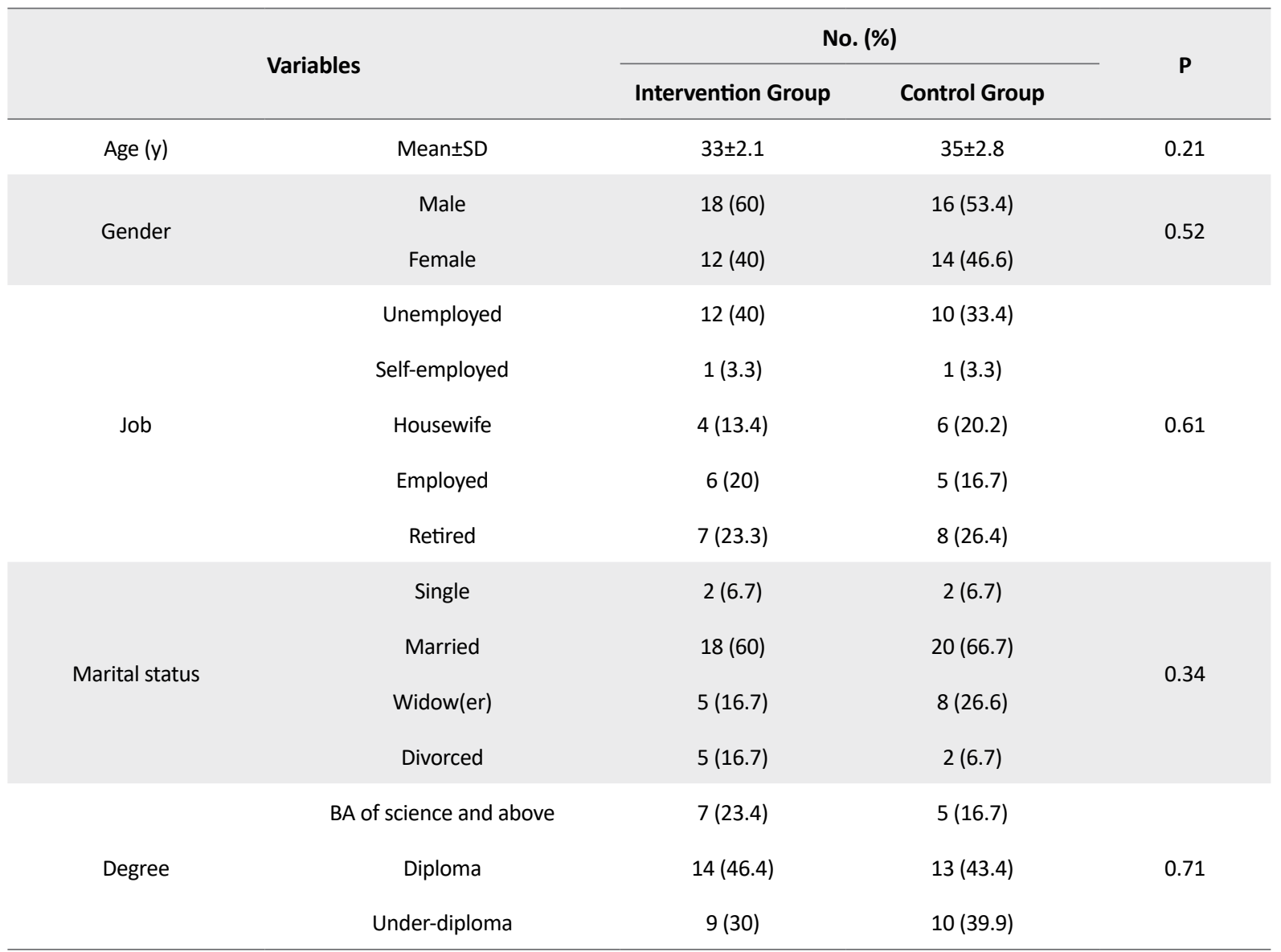


Table 2. Pre-test-post-test mean fatigue severity values in patients with MS

\begin{tabular}{|c|c|c|c|c|c|c|}
\hline \multirow{3}{*}{ Tiredness } & \multicolumn{6}{|c|}{ Mean $\pm S D$} \\
\hline & \multicolumn{3}{|c|}{ Intervention } & \multicolumn{3}{|c|}{ Control } \\
\hline & Before & After & $\mathbf{P}$ & Before & After & $\mathbf{P}$ \\
\hline Total tiredness & $4.36 \pm 1.33$ & $2.32 \pm 1.14$ & 0.001 & $4.39 \pm 1.24$ & $4.18 \pm 1.23$ & 0.31 \\
\hline Current tiredness & $6.6 \pm 1.16$ & $5.10 \pm 1.32$ & 0.001 & $6.21 \pm 1.26$ & $6.11 \pm 1.29$ & 0.24 \\
\hline $\begin{array}{l}\text { Mean tiredness } \\
\text { during the past } 24 \\
\text { hours }\end{array}$ & $4.41 \pm 1.22$ & $2.24 \pm 1.11$ & 0.01 & $4.42 \pm 1.32$ & $4.40 \pm 1.28$ & 0.32 \\
\hline $\begin{array}{l}\text { Highest tiredness } \\
\text { during the past } 24 \\
\text { hours }\end{array}$ & $7.20 \pm 1.43$ & $5.99 \pm 1.21$ & 0.001 & $7.27 \pm 1.11$ & $7.26 \pm 1.25$ & 0.22 \\
\hline
\end{tabular}

Client- Centered Nursing Care

ber was provided to the study participants, and they were requested to contact him if they had any complications or questions while performing the Benson relaxation technique. Patients in both groups were urged not to use other relaxation methods during the study. One month after the intervention, the fatigue severity was evaluated in both groups using the fatigue questionnaire.

The obtained data were analyzed in SPSS using the Chi-squared test, Independent Samples t-test, and Paired Samples t-test. Kolmogorov-Smirnov test was used to check the normality of the collected data. A Chi-squared test was applied for qualitative variables, and Paired and Independent Samples t-tests were used for the fatigue test.

\section{Results}

The achieved results suggested that the mean \pm SD age of the intervention and control groups were $33 \pm 1.2$ and $35 \pm 8.2$, respectively (Table 1 ).

Table 2 presents that the total pre-test mean score of fatigue in the intervention group was $33.1 \pm 36.4$, and af- ter the intervention, it was decreased to $14.1 \pm 2.2$. This difference was statistically significant $(\mathrm{P}=0.001)$. Furthermore, the mean score of the highest level of fatigue of the intervention group in the 24 hours before the intervention was 20.71 .43 , and after the intervention, it was $5.99 \pm 1.19$; the difference was statistically significant $(\mathrm{P}=0.001)$. However, this difference was not significant in the control group $(\mathrm{P}=0.22)$.

The difference between the mean total fatigue, current fatigue, mean fatigue over the past 24 hours, and the highest level of fatigue in the last 24 hours between the two groups was statistically significant $(\mathrm{P}<0.05)$ (Table 3 ).

Table 4 illustrates that the mean score of fatigue in the last 24 hours significantly affected the overall activity, mood, walking ability, normal work, communicating with others, and the enjoyment of life before and after the intervention in the test group $(\mathrm{P}<0.05)$. However, this difference was not statistically significant in the control group $(\mathrm{P}>0.05)$.

The collected data indicated that the mean value of the last 24 hours fatigue effect on overall activity, mood,

Table 3. Comparing the pre-test and post-test mean difference* scores of fatigue severity in patients with MS in the two groups

\begin{tabular}{|ccccc}
\hline \multirow{2}{*}{ Tiredness } & \multicolumn{2}{c}{ Mean \pm SD } & \multicolumn{2}{c}{ Statistical Tests } \\
\cline { 2 - 5 } & Intervention & Control & P & t \\
\hline Total tiredness & $2.04 \pm 0.19$ & $0.21 \pm 0.01$ & 0.001 & 2.54 \\
\hline Current tiredness & $1.06 \pm 0.16$ & $0.1 \pm 0.03$ & 0.001 & 2.10 \\
\hline Mean tiredness during past 24 hours & $2.17 \pm 0.11$ & $0.01 \pm 0.04$ & 0.001 & 2.64 \\
\hline Highest tiredness during past 24 hours & $1.21 \pm 0.22$ & $0.01 \pm 0.14$ & 0.04 & 1.98 \\
\hline
\end{tabular}

* Difference between the mean value of the beginning and the end of the study.

Client- Centered Nursing Care 
Table 4. Comparing the mean 24-hour fatigue impact

\begin{tabular}{|c|c|c|c|c|c|c|}
\hline \multirow{3}{*}{$\begin{array}{l}\text { Mean of Tiredness Impact } \\
\text { during Past } 24 \text { Hours }\end{array}$} & \multicolumn{6}{|c|}{ Mean \pm SD } \\
\hline & \multicolumn{3}{|c|}{ Intervention } & \multicolumn{3}{|c|}{ Control } \\
\hline & Pre-test & Post-test & $\mathbf{P}$ & Pre-test & Post-test & $\mathbf{P}$ \\
\hline Total activity & $4.25 \pm 1.10$ & $3.35 \pm 1.12$ & 0.002 & $4.35 \pm 1.09$ & $4.25 \pm 1.12$ & 0.32 \\
\hline Behavior & $4.35 \pm 1.23$ & $3.60 \pm 1.10$ & 0.005 & $4.35 \pm 1.14$ & $4.20 \pm 1.13$ & 0.41 \\
\hline Walking ability & $4.25 \pm 1.13$ & $3.33 \pm 1.16$ & 0.03 & $4.45 \pm 1.21$ & $4.46 \pm 1.32$ & 0.12 \\
\hline Normal work & $2.57 \pm 0.23$ & $1.93 \pm 0.12$ & 0.003 & $2.23 \pm 1.12$ & $2.23 \pm 1.09$ & 0.30 \\
\hline Communicating with others & $2.60 \pm 1.11$ & $1.56 \pm 0.10$ & 0.04 & $2.55 \pm 0.01$ & $2.53 \pm 0.11$ & 0.21 \\
\hline Enjoying life & $3.72 \pm 1.23$ & $2.65 \pm 1.21$ & 0.002 & $3.54 \pm 1.34$ & $3.51 \pm 1.30$ & 0.23 \\
\hline
\end{tabular}

Client- Centered Nursing Care

Table 5. Comparing the mean difference* of the last 24 hours fatigue

\begin{tabular}{ccccc}
\hline \multirow{2}{*}{ Tiredness } & \multicolumn{2}{c}{ Mean \pm SD } & \multicolumn{2}{c}{ Statistical Tests } \\
\cline { 2 - 5 } & Intervention Group & Control Group & P & t \\
\hline Total activity & $0.9 \pm 0.02$ & $0.1 \pm 0.03$ & 0.001 & 2.23 \\
\hline Behavior & $0.75 \pm 0.13$ & $0.15 \pm 0.01$ & 0.04 & 2.54 \\
Walking ability & $0.92 \pm 0.03$ & $0.01 \pm 0.11$ & 0.001 & 1.97 \\
\hline Normal work & $0.64 \pm 0.11$ & $0 \pm 0.03$ & 0.001 & 1.00 \\
Communicating with others & $1.04 \pm 1.01$ & $0.02 \pm 0.1$ & 0.02 & 2.87 \\
\hline Enjoying life & $1.07 \pm 0.02$ & $0.04 \pm 0.04$ & 0.001 & 2.90 \\
\hline
\end{tabular}

*Difference between the mean score of the beginning and the end of the study.

Client- Centered Nursing Care

walking ability, normal work, communicating with others, and life enjoyment before and after intervention between the two groups had a statistically significant difference $(\mathrm{P}<0.05)$ (Table 5).

\section{Discussion}

The present study findings suggested that Benson relaxation technique significantly reduced the mean value of fatigue and its effect on overall activity, mood, walking ability, normal work, communicating with others, and life enjoyment in patients with MS. Studies have revealed that Benson relaxation technique significantly affects the fatigue resulted from other chronic diseases, including renal failure (Heidari Gorji et al. 2014), and cardiovascular diseases (Seifi et al. 2018). Additionally, fatigue reduction in patients with MS is probably due to the effect of exercises on the motor and neuropsychological systems (Motl et al. 2009; Heidari Gorji et al. 2014; Seifi et al. 2018). Most studies have reported that fatigue in patients with MS was significantly reduced as a result of aerobic training (Mathiowetz et al. 2007; Stroud \& Minahan 2009; Kahraman et al. 2018). Researchers have associated this fatigue reduction on the impact of aerobic training. Nazari et al. argued that the technique of relaxation in 8 sessions of 40 minutes twice a week caused a significant difference in fatigue rate in patients with MS (Nazari et al. 2015). Some authors believe that in this technique, the relaxation of the muscles releases hormones and other chemicals in the body; therefore, this process causes physical changes, like increased blood flow in the hands and feet and reduces the severity of fatigue (Solehati and Rustina 2015; Seifi et al. 2018). Dayapoğlu and Tan documented that slowly progressive muscle relaxation techniques are effective in reducing fatigue in patients with MS (Dayapoglu and Tan 2012). Thus, the first hypothesis of the study, "Benson relaxation method affects the fatigue severity of patients with MS", was confirmed.

The present study results are inconsistent with those of some studies. For example, in a study by Rampello et al. aerobic training failed to reduce fatigue in non-affected patients (Rampello et al. 2007). The current findings were inconsistent with those of Newman et al. because the level of fatigue in patients with MS in their intervention group remained unchanged after applying aerobic exercise and relaxation (Newman et al. 2007). The reason for such data discrepancy could be related to the number of training sessions as well as the intensity of the exercise used in the programs. Possible factors that justify different results about the effect of aerobic exercises and relaxation on MS include different diets along with patient compliance, type of the pharmacotherapy, type of 
aerobic training or anaerobic exercise, endurance and resistance training, synchronous endurance and resistance training, the intensity and duration of exercises, and the method of measuring performance indicators along with the amount of attention to mental counseling of patients (Phyo et al. 2018).

The Benson relaxation technique mechanism is based on mind control and muscle relaxation. The obtained data suggested that this technique is an effective strategy for reducing fatigue in patients with MS. It is recommended that this method be applied in the care of these patients.

\section{Ethical Considerations}

\section{Compliance with ethical guidelines}

All procedures performed in this study were per the ethical standards of the institutional and national research committee and with the 1964 Helsinki Declaration and its later amendments or comparable ethical standards. This trial was registered at the Iranian Randomized Controlled Trial (Code: IRCT2014011115393N2). The study was approved by the Ethics Committee of Vice Chancellor for Research, KUMS (Ethics Code: IR.KAUMS. NUHEPM.REC.1396.31). All the study participants received explanations on the study and its objectives and signed informed consent.

\section{Funding}

This study was financially supported by the Kashan University of Medical Sciences.

\section{Authors' contributions}

Designe and obtained funding for the study: Saeed Mirhoseeni, Abouzar Mohammadi, Neda Mirbagher Ajorpaz, and Mahboobeh Rezaei; Developing the analytical plan: Neda Mirbagher Ajorpaz; Final draft of the manuscript: Mahbobeh Rezaei; Manuscript revision and data analysis: all authors.

\section{Conflict of interest}

The authors declared no conflicts of interest.

\section{References}

Abedi, F., et al. 2018. The effect of tactile-kinesthetic stimulation on growth indices of healthy neonates. Journal of Bodywork and Movement Therapies, 22(2), pp. 308-12. [DOI:10.1016/j jbmt.2017.08.005] [PMID]

Carpinella, I., et al. 2014. Quantitative assessment of upper limb motor function in Multiple Sclerosis using an instrumented Action Research Arm Test. Journal of NeuroEngineering and Rehabilitation, 11, p. 67. [DOI:10.1186/1743-0003-11-67] [PMID] [PMCID]

Chen, Y. W., et al. 2016. Reliability and validity of the brief fatigue inventory and dyspnea inventory in people with chronic obstructive pulmonary disease. Journal of Pain and Symptom Management, 52(2), pp. 298-304. [DOI:10.1016/j.jpainsymman.2016.02.018] [PMID]

Correale, J., et al. 2017. Progressive Multiple Sclerosis: From pathogenic mechanisms to treatment. Brain, 140(3), pp. 52746.

Dayapoglu, N., et al. 2012. Evaluation of the effect of progressive relaxation exercises on fatigue and sleep quality in patients with Multiple Sclerosis. Journal of Alternative and Complementary Medicine, 18(10), pp. 983-7. [DOI:10.1089/ acm.2011.0390] [PMID] [PMCID]

Etemadifar, M., et al. 2016. Early-onset Multiple Sclerosis in Isfahan, Iran: Report of the demographic and clinical features of 221 patients. Journal of Child Neurology, 31(7), pp. 932-7. [DOI:10.1177/0883073816634853] [PMID]

Etemadifar, M., et al. 2017. Multiple Sclerosis among Afghan immigrants in Isfahan, Iran. Multiple Sclerosis and Related Disorders, 13, pp. 52-57. [DOI:10.1016/j.msard.2017.02.006] [PMID]

Heidari Gorji ,M. A., et al. 2014. The efficacy of relaxation training on stress, anxiety, and pain perception in hemodialysis patients. Indian Journal of Nephrology, 24(6), pp. 356-61. [DOI:10.4103/0971-4065.132998] [PMID] [PMCID]

Heidari, S., et al. 2017. Religious practices and self-care in Iranian patients with type 2 diabetes. Journal of Religion $\mathcal{E}$ Health 56(2), pp. 683-96. [DOI:10.1007/s10943-016-0320-x] [PMID]

Heine, M., et al. 2016. Real-time assessment of fatigue in patients with Multiple Sclerosis: How does it relate to commonly used self-report fatigue questionnaires? Archives of Physical Medicine and Rehabilitation, 97(11), pp. 1887-94. [DOI:10.1016/j. apmr.2016.04.019] [PMID]

Jafari, H., et al. 2018. The effect of relaxation technique on fatigue levels after stem cell transplant. Iranian Journal of Nursing and Midwifery Research, 23(5), pp. 388-94. [DOI:10.4103/ijnmr. IJNMR_26_17] [PMID] [PMCID]

Kahraman, T., et al. 2018. Feasibility of a 6-month yoga program to improve the physical and psychosocial status of persons with Multiple Sclerosis and their family members. Explore (NY), 14(1), pp. 36-43. [DOI:10.1016/j.explore.2017.07.006] [PMID]

Kamińska, J., et al. 2017. Multiple Sclerosis-etiology and diagnostic potential. Advances in Hygiene and Experimental Medicine (Online), 71(0), pp. 551-63. [DOI:10.5604/01.3001.0010.3836] [PMID]

Mathiowetz, V. G., et al. 2007. One-year follow-up to a randomized controlled trial of an energy conservation course for persons with Multiple Sclerosis. International Journal of Rehabilitation Research, 30(4), pp. 305-13. [DOI:10.1097/ MRR.0b013e3282f14434] [PMID] 
Montalban, X., et al. 2018. ECTRIMS/EAN guideline on the pharmacological treatment of people with Multiple Sclerosis. Multiple Sclerosis Journal, 24(2), pp. 96-120.

Motl, R. W, et al. 2009. Physical activity and quality of life in Multiple Sclerosis: Intermediary roles of disability, fatigue, mood, pain, self-efficacy and social support. Psychology, Health $\mathcal{E}$ Medicine, 14(1), pp. 111-24. [DOI:10.1080/13548500802241902] [PMID] [PMCID]

Mowla, F., et al. 2017. Contribution of Benson's relaxation technique and brief psycho-educational intervention on quality of life of primary caregivers of Iranian children with chronic diseases. Journal of Pediatric Nursing, 35, pp. 65-71. [DOI:10.1016/j.pedn.2017.02.037] [PMID]

Nazari, F., et al. 2015. Comparing the effects of reflexology and relaxation on fatigue in women with Multiple Sclerosis. Iranian Journal of Nursing and Midwifery Research, 20(2), pp. 2004. [PMCID] [PMID]

Newman, M. A., et al. 2007. Can aerobic treadmill training reduce the effort of walking and fatigue in people with Multiple Sclerosis: A pilot study. Multiple Sclerosis Journal, 13(1), pp. 113-9. [DOI:10.1177/1352458506071169] [PMID]

Niino, M., et al. 2014. Apathy/depression, but not subjective fatigue, is related with cognitive dysfunction in patients with Multiple Sclerosis. BMC Neurology, 14, p. 3. [DOI:10.1186/1471-2377-14-3] [PMID] [PMCID]

Nunnari, D., et al. 2015. Impact of depression, fatigue, and global measure of cortical volume on cognitive impairment in Multiple Sclerosis. BioMed Research International, 2015, p. 519785. [DOI:10.1155/2015/519785] [PMID] [PMCID]

Ontaneda, D., et al. 2017. Progressive Multiple Sclerosis: Prospects for disease therapy, repair, and restoration of function. Lancet, 389(10076), pp. 1357-66. [DOI:10.1016/S01406736(16)31320-4]

Phyo, A. Z. Z., et al. 2018. The efficacy of psychological interventions for managing fatigue in people with Multiple Sclerosis: A systematic review and meta-analysis. Frontiers in Neurology, 9, p. 149. [DOI:10.3389/fneur.2018.00149] [PMID] [PMCID]

Pocock, S. J., 1983. Clinical Trials: A Practical Approach. Hoboken, New Jersey: John Wiley \& Sons.

Rahimimoghadam, Z., et al. 2017. Effects of Pilates exercise on general health of hemodialysis patients. Journal of Bodywork and Movement Therapies, 21(1), pp. 86-92. [DOI:10.1016/j. jbmt.2016.05.012] [PMID]

Rahimimoghadam, Z., et al. 2019. Pilates exercises and quality of life of patients with chronic kidney disease. Complementary Therapies in Clinical Practice, 34, pp. 35-40. [DOI:10.1016/j. ctcp.2018.10.017] [PMID]

Rambod, M., et al. 2013. The effect of Benson's relaxation technique on the quality of sleep of Iranian hemodialysis patients: A randomized trial. Complementary Therapies in Medicine, 21(6), pp. 577-84. [DOI:10.1016/j.ctim.2013.08.009] [PMID]

Rampello, A., et al. 2007. Effect of aerobic training on walking capacity and maximal exercise tolerance in patients with Multiple Sclerosis: A randomized crossover controlled study. Physical Therapy, 87(5), pp. 545-55. [DOI:10.2522/pti.20060085] [PMID]
Rooney, S., et al. 2019. Prevalence of fatigue and its association with clinical features in progressive and non-progressive forms of Multiple Sclerosis. Multiple Sclerosis and Related Disorders, 28, pp. 276-82. [DOI:10.1016/j.msard.2019.01.011] [PMID]

Seifi, L., et al. 2018. Comparison of the effects of Benson muscle relaxation and nature sounds on the fatigue in patients with heart failure: A randomized controlled clinical trial Holistic Nursing Practice, 32(1), pp. 27-34. [DOI:10.1097/ HNP.0000000000000242] [PMID]

Solehati, T., Rustina, Y,. 2015. Benson relaxation technique in reducing pain intensity in women after cesarean section. $A n-$ esthesiology and Pain Medicine, 5(3), p. e22236. [DOI:10.5812/ aapm.22236v2] [PMID] [PMCID]

Stroud, N. \& Minahan, CL., 2009. The impact of regular physical activity on fatigue, depression and quality of life in persons with Multiple Sclerosis. Health and Quality of Life Outcomes, 7 , p. 68. [DOI:10.1186/1477-7525-7-68] [PMID] [PMCID]

Van Kessel, K., et al. 2008. A randomized controlled trial of cognitive behavior therapy for Multiple Sclerosis fatigue Psychosomatic Medicine, 70(2), pp. 205-13. [DOI:10.1097/ PSY.0b013e3181643065] [PMID] 